\title{
Junction region of EWS-FLI1 fusion protein has a dominant negative effect in Ewing's Sarcoma in vitro
}

Babu Jully, Ramshankar Vijayalakshmi ${ }^{\dagger}$, Gopisetty Gopal ${ }^{\dagger}$, Kesavan Sabitha and Thangarajan Rajkumar ${ }^{*}$

\begin{abstract}
Background: Ewing's sarcoma is a malignancy characterized by a specific 11:22 chromosomal translocation which generates a novel EWS-FLI1 fusion protein functioning as an aberrant transcription factor. In the present study, we have further characterized the junction region of the EWS-FLI1 fusion protein.

Methods: In-silico model of EWS-FLI1 fusion protein was analysed for ligand binding sites, and a putative region (amino acid (aa) 251-343 of the type 1 fusion protein) in the vicinity of the fusion junction was cloned and expressed using bacterial expression. The recombinant protein was characterized by Circular Dichroism (CD). We then expressed aa 251-280 ectopically in Ewing's sarcoma cell-line and its effect on cell proliferation, tumorigenicity and expression of EWS-FLI1 target genes were analysed.
\end{abstract}

Results: Our modelling analysis indicated that Junction region (aa 251-343) encompasses potential ligand biding sites in the EWS-FLI1 protein and when expressed in bacteria was present as soluble form. Ectopically expressing this region in Ewing's sarcoma cells inhibited tumorigenicity, and EWS-FLI1 target genes indicating a dominant negative biological effect.

Conclusions: Junction region can be exploited further as target for drug development in future to specifically target EWS-FLI1 in Ewing's Sarcoma.

\section{Background}

Ewing's sarcoma is a highly malignant bone and soft tissue tumor occurring in children and young adults. More than $85 \%$ of the Ewing's sarcoma family of tumours (ESFT) patients present with a balanced $\mathrm{t}(11: 22)$ (q24; q12) chromosomal translocation [1,2]. This reciprocal translocation generates a novel in frame fusion gene with a unique junctional region between sequences which encode the N-terminus of the RNA binding protein EWS from chromosome 22 and the C-terminus of FLI1 transcription factor on chromosome 11 [3,4]. Several evidences have shown EWS-FLI1 as a well described oncogene and with depletion of this gene product resulting in inhibition of ESFT growth. EWS-FLI1 fusion protein therefore is a validated tumor target functioning as an aberrant transcription factor [4,5]. Transforming

\footnotetext{
* Correspondence: drtrajkumar@gmail.com

${ }^{\dagger}$ Equal contributors

Department of Molecular Oncology, Cancer Institute (WIA), 38, Sardar Patel Road, Chennai 600036, India
}

activity of EWS-FLI1 requires both the EWS portion of the fusion protein which contributes to transactivation and the ETS domain (FLI1 portion) which mediates sequence-specific DNA binding [6-8].

Structure of EWS-FLI1 is not available in the PDB and it is an intrinsically disordered Protein (IDP). These kind of proteins are insoluble, unstructured and do not have specific Ramachandran angles in the protein backbone and show polymorphism in bound state $[9,10]$.

In this study, we looked at EWS-FLI1 protein structure using modelling tool and bioinformatics tools to analyze potential structure in-silico. Our analysis indicated potential ligand binding sites which encompass the junction region of the EWS-FLI1 protein and that the region was likely to have a structure indicated by alpha helical and beta pleated structures. The junction region (aa 251-343) containing type 1 fusion residues was expressed and purified and subjected to circular dichroism (CD) analysis. Finally our analysis of the biological effects of ectopically expressing junction region on 
expression of EWS-FLI1 target genes, and proliferation of Ewing's sarcoma cells in-vitro indicates a dominant negative function for the junction region.

\section{Methods \\ RNA Extraction and cDNA preparation}

The study was approved by the Cancer Institute Ethics Committee. RNA was isolated from biopsy sample from a patient diagnosed with Ewing's Sarcoma after an informed consent. RNA was qualitatively and quantitatively assessed on $1.25 \%$ agarose gel and by spectrophotometer. cDNA was synthesized using Superscript II (Invitrogen) as per manufacturer's instructions and $\beta$ actin amplification was done to check its quality. Full length EWS-FLI1 Type1 fusion gene was amplified. The PCR product was purified and sequenced using ABI 310 Genetic analyzer. This sequence was submitted in GenBank [GenBank: ACA62796].

\section{In-silico analysis of EWS-FLI1 protein using prime and SiteMap}

Prime (Version 3.0, Schrodinger, LLC, New York) was used to build EWS-FLI1 structure. The OPLS2000 allatom force field was used for energy scoring of proteins. Surface Generalized Born (SGB) continuum solvation model, was used for treating solvation effects; and side chain rotomer and backbone dihedral libraries derived from PDB non-redundant structures were used for building backbone and side chains. The modelled structure was imported and corrections were carried out by Protein Preparation wizard of Schrodinger, where hydrogens were added automatically and refinement of the structure was done. EWS-FLI1 protein was assessed for putative ligand binding sites using SiteMap. The programme highlights regions within the protein suitable for occupancy by hydrophobic groups or by ligand hydrogen-bond donors, acceptors, or metal-binding functionality. SiteScore, the scoring function was used to assess a site's propensity for ligand binding, and rank possible binding sites.

\section{Cloning and expression of EWS-FLI1}

The sequenced EWS-FLI1 Type 1 fusion gene was cloned in $\mathrm{pET}$ 102/D-TOPO vector (Invitrogen) using the primer set PET 102/D-TOPO Forward. 5'CAC CATGGCGTCCACGGATT3' and Reverse. 5'GTAG TAGCTGCCTAAGTGTGA 3'. Full length EWS-FLI1 type 1 c-DNA was cloned into pCR2.1 using TA cloning method. The insert was then subcloned into pGEX-KG a GST fusion vector to be expressed as GST fusion protein. Ligation mixtures of EWS-FLI1 in pET 102/D TOPO and pGEX-KG were used to transform E.coli TOP 10, chemically competent cells and selected in ampicillin $(100 \mu \mathrm{g} / \mathrm{ml})$ containing medium. The positive clones were determined by colony PCR and sequencing. Full length EWS-FLI1 was cloned into pGEX-KG and pET 102/D-TOPO vector were expressed in BL21-CodonPlus (DE3)-RP competent cells (Stratagene, La Jolla, CA) containing extra copies of ArgU and ProL genes to overcome the codon bias [11]. Protein production was initiated by adding $0.4 \mathrm{mM}$ isopropyl $\beta-\mathrm{D}-1$ - thio galactopyranoside (IPTG), and bacteria were cultured for an additional 3-4 $\mathrm{h}$ at $20^{\circ} \mathrm{C}$.

\section{Immunoblotting}

Lysates from Bacteria cells expressing Thioredoxin (Trx)-EWS-FLI1-His tagged protein, whole cell lysates from MCF-7, EWS502 cells stably expressing pCDNA/ FLAG and pCDNA/FLAG/Junction (aa 251-280) constructs were used for immunoblot analysis. The antibodies used were anti-FLI1 (C-19) antibody (Santa Cruz Biotechnology, Inc., Santa Cruz, CA, USA), E Cadherin (abcam, Cambridge, UK), Vimentin (abcam, Cambridge, UK), Beta Actin (Sigma, Saint Louis, MI, USA). Bacteria cells expressing Thioredoxin (Trx)-EWS-FLI1-His tagged protein, suspended in native lysis buffer $(20 \mathrm{mM}$ Tris $\mathrm{pH}$ 8, $500 \mathrm{mM} \mathrm{NaCl}, 0.1 \% \mathrm{NP} 40$, Lysozyme $1 \mathrm{mg} / \mathrm{ml}$ ), subjected to lysis by sonication and centrifuged at 12000 rpm. The supernatant (soluble) and pellet (insoluble) fraction were analysed in SDS PAGE (10\%). Lysates from MCF-7, and EWS502 cells stably expressing pCDNA/ FLAG or pCDNA/FLAG/Junction (aa 251-280) constructs were prepared in RIPA buffer (1\% Nonidet P-40, $1 \%$ sodium deoxycholate, $0.1 \%$ SDS, 0.15M Sodium chloride, $0.05 \mathrm{M}$ Tris.Cl, pH8.0). 50 micro grams of the whole cell lysate was used for the analysis. Gels were transferred onto poly vinylidene difluoride (PVDF) membrane (Millipore, Billerica, MA, USA). This filter was blocked with 5\% non-fat milk in TBS-T (20 mM Tris- $\mathrm{HCl}$, $\mathrm{pH}$ 7.5, $150 \mathrm{mM} \mathrm{NaCl}, 0.1 \%$ Tween 20) for $1 \mathrm{hr}$ at room temperature and then probed with respective primary and secondary antibody. The blot was incubated and visualized with enhanced chemiluminescence (ECL) solution (GE Healthcare Life Sciences, Buckinghamshire, UK) according to the manufacturer's instructions.

\section{Purification of recombinant EWS-FLI1}

Trx-Full length EWS-FLI1-6xHis tagged Protein was purified by Ni-NTA immobilized metal-ion affinity chromatography (IMAC) under denaturing conditions. Lysis/ Equilibration buffer/wash buffer compositions: $50 \mathrm{mM}$ $\mathrm{Na}_{3} \mathrm{PO}_{4}$ (pH 7.8) containing $500 \mathrm{mM} \mathrm{NaCl}, 20 \mathrm{mM}$ Tris $\mathrm{HCl}, 6 \mathrm{M}$ Urea and Elution buffer: wash buffer containing $200 \mathrm{mM}$ imidazole. Initially, precharged metal chelating columns were washed twice with $10 \mathrm{~mL}$ of distilled water, and equilibrated in $10 \mathrm{~mL}$ of equilibration buffer. Cell pellet corresponding to $100 \mathrm{~mL}$ of bacterial culture was re-suspended in $10 \mathrm{~mL}$ of lysis buffer. Debris was 
removed by centrifugation at $4000 \mathrm{~g}$ for $10 \mathrm{~min}$ at $4^{\circ} \mathrm{C}$. The supernatant was applied to Ni-NTA affinity column (Invitrogen, Carlsbad, CA, USA ) and incubated at room temperature for 30 minutes with constant rocking [12]. Column was washed three times and protein was then eluted from the column using $200 \mathrm{mM}$ imidazole. Aliquots $(1 \mathrm{~mL})$ were collected and a portion of which was then applied to $10 \%$ polyacrylamide gel for Coomassie staining and immunoblotting.

\section{Tryptic mapping and mass spectrometry}

Mass spectrometry of the purified EWS-FLI1 was performed at the Mass Spectroscopy Facility at The Centre for Genomics Research, New Delhi, India.

\section{Fold index plot}

Amino acid sequence of EWS-FLI1 (submitted in GenBank) was assessed to identify the ordered and disordered region within the protein using Fold Index predictor available as a graphic web server [13,14].

\section{Purification of soluble junction construct}

Junction (aa 251 - 343) region was cloned in pET 102/ D-TOPO vector (Invitrogen Carlsbad, CA, USA) using primer set Junction (aa 251 - 343) Forward. 5'CACCC CAAGTCAATATAGCAACAGAGC 3, and Junction (aa 251 - 343) Reverse. 5'GGCGTTGGCGCTGTCGGAG 3' and expressed in BL21-CodonPlus (DE3)-RP competent cells. Expression of Trx-Junction (aa 251-343) -6xHis tagged protein was confirmed by immunoblotting using anti-thio antibody and horseradish peroxidaseconjugated anti-mouse antibodies as primary and secondary antibody, respectively. Protein was purified by Ni-NTA immobilized metal-ion affinity chromatography (IMAC) under native condition. Buffer compositions were: Lysis/Binding/Equilibration buffer 50 $\mathrm{mM}$ sodium phosphate buffer, $300 \mathrm{mM} \mathrm{NaCl}, 5 \mathrm{mM}$ imidazole, $0.1 \% \mathrm{NP}-40 \mathrm{pH} 8.0$. Wash (W1) $50 \mathrm{mM}$ sodium phosphate buffer, $300 \mathrm{mM} \mathrm{NaCl}, 20 \mathrm{mM}$ imidazole, pH 8.0. Wash (W2) $50 \mathrm{mM}$ sodium phosphate buffer, $300 \mathrm{mM} \mathrm{NaCl}, 50 \mathrm{mM}$ imidazole, pH 8.0. Elution buffer $50 \mathrm{mM}$ sodium phosphate buffer, $300 \mathrm{mM}$ $\mathrm{NaCl}, 200 \mathrm{mM}$ imidazole, $\mathrm{pH}$ 8.0.

\section{Dialysis and circular dichroism}

Affinity purified Junction protein (aa 251-343) in buffer containing $50 \mathrm{mM}$ sodium phosphate buffer, $300 \mathrm{mM}$ $\mathrm{NaCl}, 200 \mathrm{mM}$ imidazole, $\mathrm{pH} 8.0$ was concentrated using Centricon concentrator (EMD Millipore, Billerica, MA, USA) with a $10 \mathrm{kDa}$ cut off and dialyzed against $10 \mathrm{mM}$ sodium phosphate buffer, $\mathrm{pH}$ 8.0. The concentrated protein $(2 \mathrm{mg} / \mathrm{ml})$ was subjected to circular dichroism.

\section{Expression of short junction region (aa 251 to aa 280) in Ewing Sarcoma cells}

The short junction construct, a 30 amino acid containing peptide (aa 251 to aa 280) which overlaps EWS-FLI1 junction region was cloned into pcDNA3.1/FLAG vector at EcoRV and XhoI restriction sites using the primer set (aa 251 to aa 280) Forward. 5'GATATCCCAAGTCAAA TAACCCAACAGAG 3' and (aa 251 to aa 280) Reverse 5'CTCGAGCTACATGTTATTGCCCCA 3'. EWS502 Ewing's sarcoma cell line was grown in RPMI-1640 supplemented with $10 \%$ fetal calf serum. $5 \mu \mathrm{g}$ of pCDNA3.1/ FLAG-junction and pCDNA3.1/FLAG plasmid alone were transfected separately into EWS502 cell line (harbouring EWS-FLI1 type1 fusion gene) using Fugene (Roche, Molecular Biochemicals, Indianapolis, IN, USA) transfection kit as per the manufacturer's instructions. The transfected cell line was selected in $100 \mu \mathrm{g} / \mathrm{ml}$ hygromycin and maintained in presence of hygromycin. Single clone of transfected cells was isolated by serial dilution. Expression of Flag tagged junction construct in transfected EWS502 cell line was confirmed by western blot.

\section{Colony forming assay}

EWS502 pCDNA/FLAG alone and EWS502 pCDNA/ FLAG/junction (aa 251-280) cells were plated in agar in duplicates at density of 5000 cells per well in a 6 well culture plate. Bottom layer was prepared with $0.5 \%$ agar and top layer with $0.2 \%$ agar. Culture plates were incubated at $37^{\circ} \mathrm{C}$ and $5 \% \mathrm{CO} 2$ in a humidified atmosphere. Colonies were enumerated after 3 weeks of growth. All the experiments were repeated at least twice.

\section{Real-Time quantitative PCR}

Real time quantitation of EWS-FLI1 modulated targets NROB1, NKX2.2, GLI1, Cyclin D1, c-MYC, EZH2, TGFßIIR, KFL2 and EMT markers E-Cadherin, Vimentin, Slug, N-Cadherin and Fibronectin was done using SYBR green master mix (Fermentas GMBH, Germany) as per manufacturer's instructions. Three individual clones of the junction construct (aa 251-280) transfected EWS502 were analyzed for Real time quantitation. Each sample was assessed in triplicate to ensure reproducibility of the quantitative measurements. GAPDH expression was evaluated for each sample as a control for total RNA. The experiments were repeated at least twice.

\section{Results}

Junction region (aa 251 to aa 343) encompasses potential ligand binding sites in the EWS-FLI1 protein

Since the crystal structure of EWS-FLI1 protein was not available we chose to study the putative structure of the protein modelled using an proprietary algorithm insilico. The protein sequence of EWS-FLI1 accession number ACA62796 was used for modelling. The modelled 
structure of EWS-FLI1 indicated the presence of alpha helices (30-37, 88-94, 116-120, 124-127, 254-262, 264268, 326-334, 401-412, 458-465), beta sheets (12-15, 54-57, 105-109, 147,148, 352,353, 377-380, 387$389,425,426)$ and loops (Figure 1A). We examined the protein structure for potential ligand association sites. Sites which received a score greater than 1.0 indicate that they are likely to bind with ligands. Around 5 sites were identified, of which four sites had a site score greater than 1 and encompassed the junction region amino acids (Table 1). Sites 1 and 6 are depicted in Figure 1B and C. Since junction region amino acids were predicted to be part of the potential ligand binding sites we were interested in further understanding the structure in the vicinity of the junction region. Analysis of the structure of junction region extending between aa 251 and aa 343 comprising of both EWS region and FLI1 potion indicated the presence of alpha helical regions (Figure 1D).

Further when analyzed using Fold index tool, the region in the FLI1 portion of the junction indicated a stable region which however did not extend into the EWS portion (Figure 2). Since our modeling studies indicated a functional role as a putative ligand binding region and presence of structured regions in the junction region (aa 251-343) we proceeded to express and purify the junction region.

Full Length protein is insoluble where as the junction region (aa 251 to aa 343 ) is soluble

To start with we expressed the full length recombinant EWS-FLI1 protein with a thioredoxin (Trx) tag in the amino terminus and 6xHis-Tag in the carboxy terminus was expressed in BL21-CodonPlus (DE3)-RP cells. A band consistent with EWS-FLI1 (68 kDa) was observed in the insoluble fraction (Figure 3A) which was confirmed by western blot using Anti FLI1 antibody (Figure 3B). The insoluble fraction of EWS-FLI1 was purified using Ni-NTA purification system in the denaturing condition (Figure 3C). Similarly, GST-EWS-FLI1 could not be taken further for native affinity purification because of its insolubility. The junction region aa 251-343 identified from our modeling studies to be relatively structured was cloned and expressed in a similar manner to the full length protein. The junction region was expressed in soluble fraction of the lysate. Expression of soluble junction region (aa 251-343) was confirmed by Western blot (Figure 4A). Junction region was purified in native condition using Ni-NTA purification system (Figure 4B), concentrated and circular dichroism analysis was performed. The $\mathrm{CD}$ readings were taken for the wavelength range of 190-350nm showed highly coiled form (random)- 48\%, Beta sheets- $42 \%$ and alpha helix $10 \%$ (Figure $4 \mathrm{C}$ ) indicating the presence of structured regions in the protein.

\section{Ectopic expression of junction construct (aa 251 to aa 280) represses tumorigenicity and alters the expression of EWS-FLI1 target genes}

In order to explore the biological effects of junction region a smaller portion comprising of thirty amino acids (251 to 280) was ectopically expressed in EWS502 Ewing's
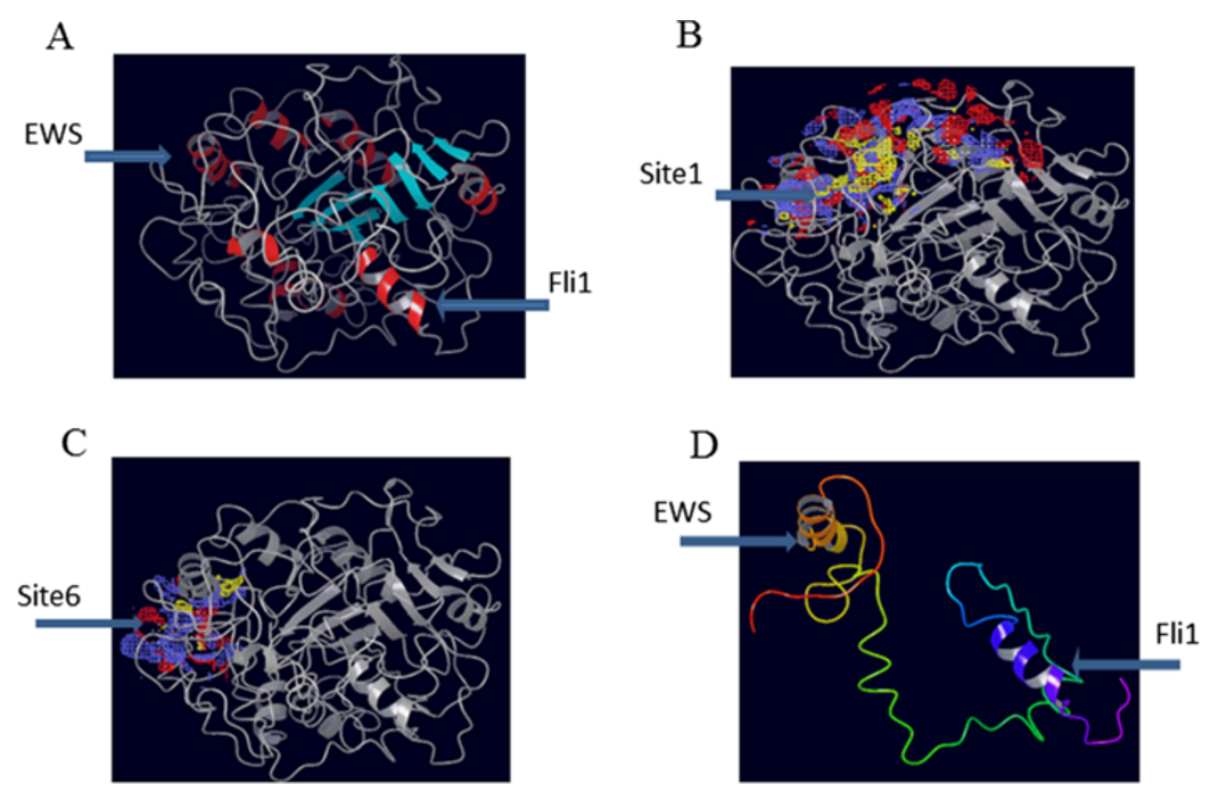

Figure 1 In-silico structure of EWS-FLI1 protein and putative small molecule binding regions. A, In-silico structure of EWS-FLI1 protein. Figure $1 \mathbf{B}$ and $\mathbf{C}$ model structures denoting the small molecule binding regions. Figure $1 \mathbf{D}$ the structure of the region form a.a.251- a.a.343 in the vicinity of the junction region of EWS-FLI1 protein. 
Table 1 Putative small molecule binding sites in EWS-FLI1 protein

\begin{tabular}{|c|c|c|}
\hline SITE & Residue Number & Score \\
\hline 4 & 21,156-167,170,173,176-196,203-207,218- 223 247,248, 317-327, 389, 391, 394,447-450,454,498 & 1.151 \\
\hline 3 & $\begin{array}{l}109-112,117,120,121,124,125,128,129,146-148,150-153,298305,307,308,310,315,317,324,325,327,328,331,332,335,345,347,380,382,383,385- \\
389,481-490\end{array}$ & 1.077 \\
\hline 2 & $12-16,54-64,66-69,81-86,89-98,100-103,105,107,109,112,119-124,127,128,132-146,272,276,290,296,311$ & 1.047 \\
\hline 6 & $25,65,71-75,77,209,213-217, \mathbf{2 3 6 - 2 4 7 , 2 5 0 , 2 5 4 - 2 6 4 , 2 7 8 - 2 8 6}$ & 1.043 \\
\hline 5 & 1,14-16,29,31,32,35,36,41-44,49,50,54-59,66,67,90,92-97,261-264,267-272 & 1.032 \\
\hline 9 & $150,151,158-160,315,317-329,350,377-380,387-391,456,461,489-492$ & 1.026 \\
\hline 1 & $22,25-34,36-40,163,188,190-211,214,216-218, \mathbf{2 4 6 - 2 5 4 , 2 5 7 - 2 5 9 , ~ 2 6 1 , 2 6 2 , 2 6 4 , 2 7 9 - 2 8 2 , 3 1 8 , 3 5 2 , 3 6 5 - 3 6 9 , 3 7 7 , 3 7 8 , 3 9 1 - 4 0 1 , 4 3 6 , 4 3 9 - 4 5 0 , 4 5 4 ~}$ & 1.007 \\
\hline 8 & $179-181,184,206-208,216,219-223,229-237, \mathbf{2 4 0 , 2 4 4 , 2 4 6 , 2 8 5 , 2 8 8 , 2 8 9}$ & 0.944 \\
\hline 7 & $11,104,106,144-147,307-314,374,383-388,410-413,476,479-482,484$ & 0.948 \\
\hline 10 & $61-63,80-86,89,118,119,223,234,235,288-296$ & 0.777 \\
\hline
\end{tabular}

The table lists the binding sites along with the amino acid residues which form the sites. Residues numbered in bold denote the region in the vicinity of the junction of EWS-FLI1 protein.

Sarcoma cell line. The stable expression of the junction region was confirmed by immunoblotting for the presence of FLAG tagged junction region. (Figure 5A). The over expression was further confirmed by real-time PCR analysis which indicated an $\log (2)$ fold increase of 5.96 in over expressing cells (Figure 5B). When soft agar assay was performed on these cells we found a marked reduction in the colony forming propensity of EWS502 cells in the

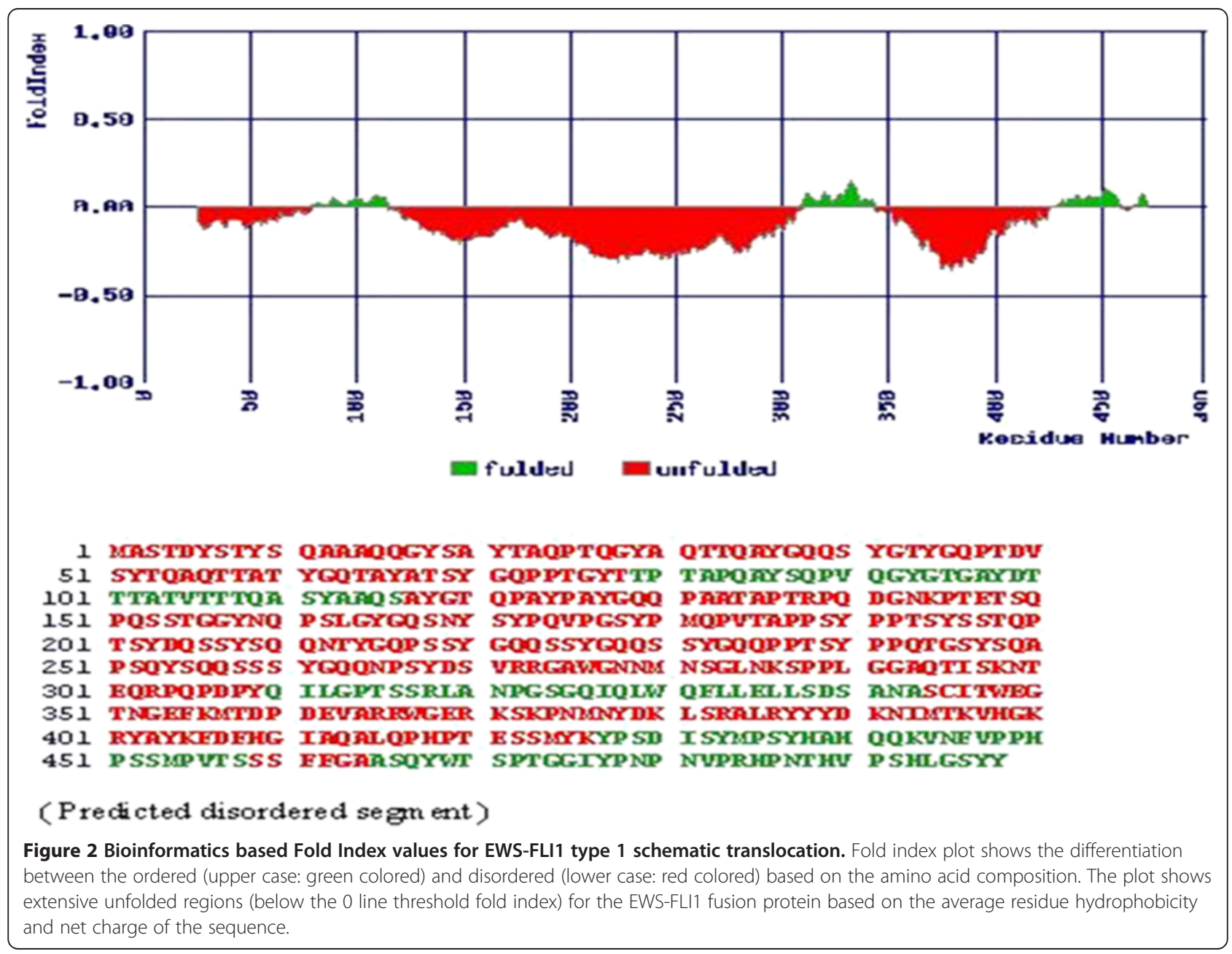




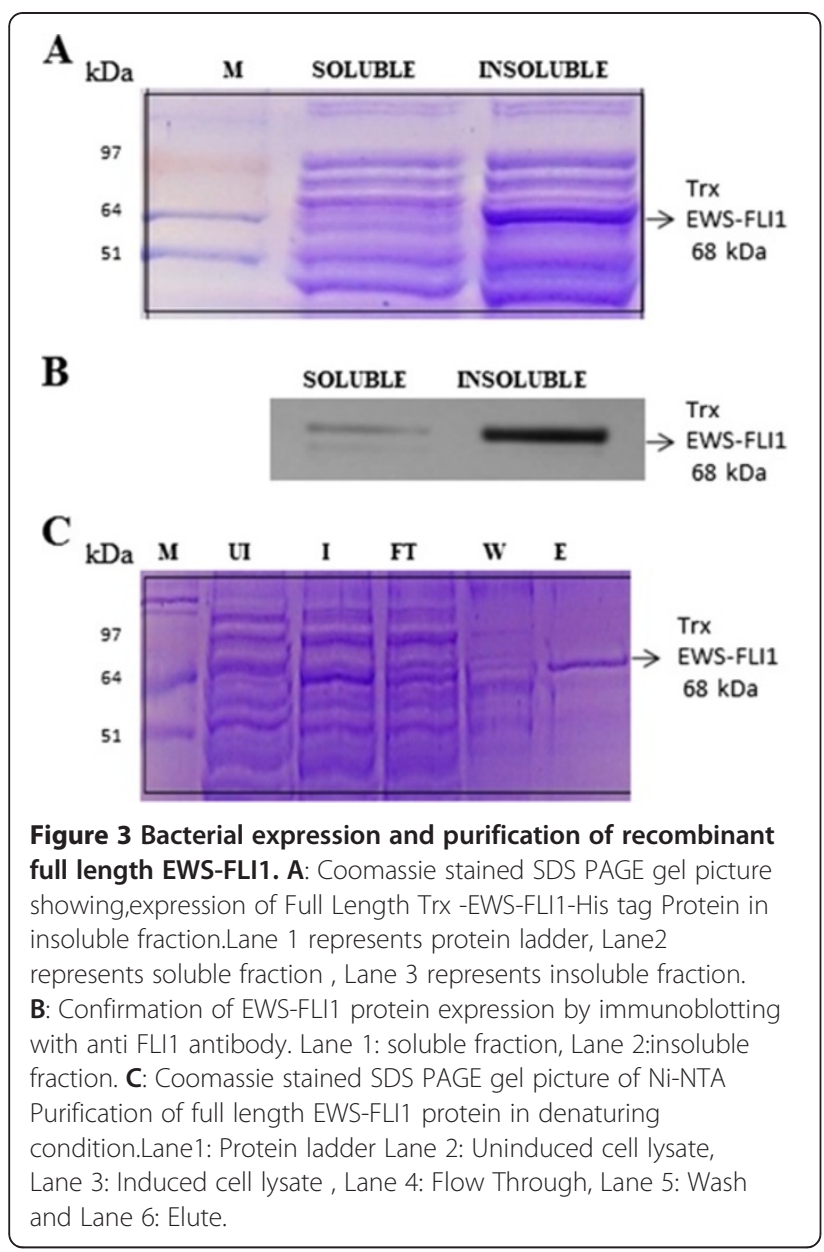

presence of junction construct, p-value $<0.001$ (Figure 5C), this indicated that the over expression of junction construct could inhibit anchorage independence and hence tumorigenicity of Ewing sarcoma cells.

Junction construct transfected EWS502 cells underwent marked morphologic changes in vitro, adopting a round, rather than the elongated phenotype of empty vector transfected counterpart (Figure 5D).

Marked decrease in the colony forming ability observed led us to study the expression of the known EWS-FLI1 regulated targets. Real time quantitative RT-PCR analysis of EWS-FLI1 up-regulated target genes were found to be down-regulated in junction construct (a.a. 251-280) transfected cell line - GLI1 by 2.47 fold, NKX2.2 by 2.3 fold, NROB1 by 3.15 fold, Cyclin D1 by 2.4 fold, c-MYC 1.69 by fold, and EZH2 by 1.48 fold compared to pcDNA-EWS502 vector control cell line (Figure 6A). Known EWS-FLI1 downregulated targets like TGF $\beta$ RII, and KLF2 showed up-regulation of 11.9 fold and 4.85 fold respectively in junction construct transfected EWS502 cells, compared to vector control.
Epithelial to mesenchymal transition (EMT) marker genes expression is repressed in the junction construct (aa 251 to aa 280) over-expressing cells

The suppression of tumorigenic potential by the junction construct (aa 251-280) transfected cells also led us to study the effect on expression of EMT markers.

Mesenchymal markers were found to be down regulated (Figure 6B) in junction construct transfected EWS502 cell line. Fibronectin (6.26 fold), Vimentin (1.75 fold) and N Cadherin (5.69 fold) were down regulated compared to vector control. EMT promoting gene Slug was also found to be down regulated by 2.1 fold (Figure 6B).

Epithelial marker E-Cadherin showed 4 fold up regulation in junction construct transfected EWS502 (compared to vector control). Immunoblotting was performed to assess the protein expression levels of E-Cadherin and Vimentin relative to pCDNA/FLAG expressing cells. Cell lysate from MCF-7 cells were used as positive control for the expression of E-Cadherin and Vimentin and Beta Actin expression as a control for protein loading. The analysis showed a distinct increased expression of E Cadherin and repression of Vimentin expression in junction construct (aa 251-280) expressing cells (Figure 6C and D). The expression levels assessed for EMT marker genes further indicate the potential of the junction construct (aa 251-280) to repress tumorigenic properties Ewing's sarcoma cells.

\section{Discussion}

EWS-FLI1 is an ideal therapeutic target protein in Ewing's sarcoma due to its causative role in the process of tumorigenesis $[15,16]$. Structural and functional characterization of EWS-FLI1 is therefore important to specifically target this protein which is very likely to benefit patients with Ewing's sarcoma. Deeper understanding of its sequence structure relationships promises to enable design of novel therapeutic molecules.

EWS-FLI1 is an intrinsically disordered protein and structural property of this protein is less characterized due to its insolubility. In the present study, we modelled the EWS-FLI1 of type 1 fusion type in-silico and identified potential ligand binding sites. The insolubility of the full length EWS-FLI1 could not be overcome despite the widely used solubility tags like Trx and GST. Recovery of the protein in denaturing conditions and refolding was not attempted in this study because studies done previously show, purified EWS-FLI1 and refolded EWS-FLI1 exhibiting differences in orientation of aromatic side chains on exposure to solvent [17]. We therefore identified the ordered region within the EWS-FLI1 structure which had the junction region specific to EWS-FLI1 type 1 and characterized it by circular dichroism (CD).

The junction region of any oncogenic fusion protein is unique for targeting and we, for the first time found the junction region (aa 251-343) of EWS-FLI1 fusion protein 


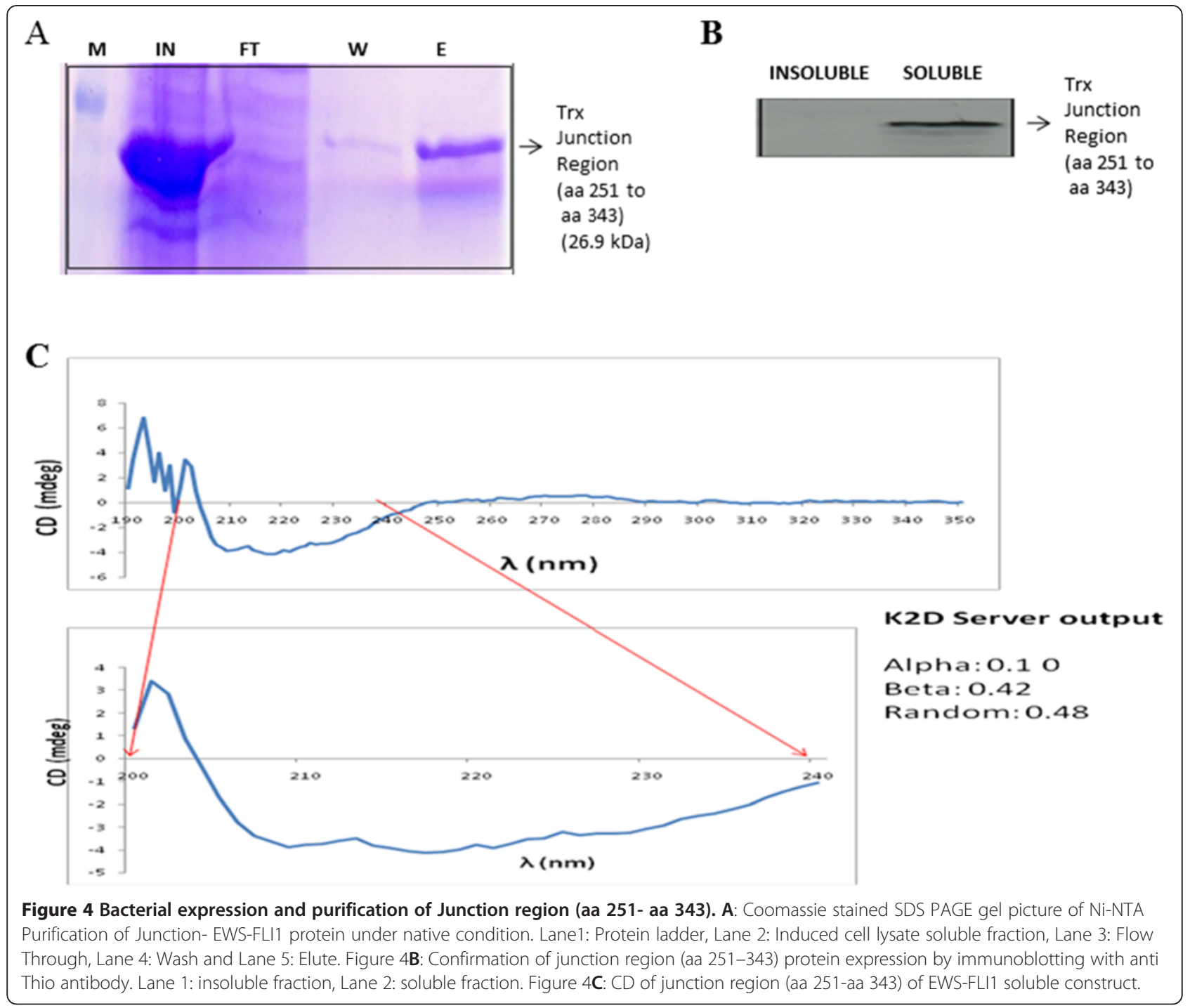

to be soluble. Protein solubility under physiological conditions is a prerequisite for drug development, though previous reports [17] have shown the insolubility of the EWS-FLI1, our findings show that the junction region can be obtained in the soluble form and can be used for further structural exploration, with a potential to be a drug target.

Further work needs to be carried out to understand the transition from a disordered to an ordered state by performing $\mathrm{CD}$ experiments with titrations against possible interacting proteins or other molecules (for e.g. Promoter Oligonucleotides) regulated by this fusion protein [18-20]. This could be useful in obtaining a crystal structure for the junction region of this fusion protein which would enable identifying a unique target.

As per our knowledge we are the first to describe the dominant negative behaviour of the junction construct (aa 251 - 280). Previous study [7] with EWS-FLI1 deletion mutants showed that both EWS and FLI1 domains are necessary for transformation and deletion of either the EWS domain or the FLI1 DNA-binding domain totally abrogates the transforming ability. In our study we found that the junction region behaved in a dominant negative manner by suppressing tumorigenicity of EWS502 Ewing's Sarcoma cell line in the soft agar assay. This attenuation could be an indirect effect due to competition for the molecular targets between the wild type EWSFLI1 present in EWS502 and junction region protein which was ectopically overexpressed, or junction region protein interacting with the full length wild type EWS-FLI1 rendering inactive complexes between the two forms of protein.

Because previous studies [21], have suggested that transcriptional activation is critical to the function of EWS-FLI1 as an oncoprotein, we focused our efforts on genes that were up regulated by the fusion protein. Quantitative Real Time PCR was done to check the expression level of some of the well known EWS-FLI1 up 


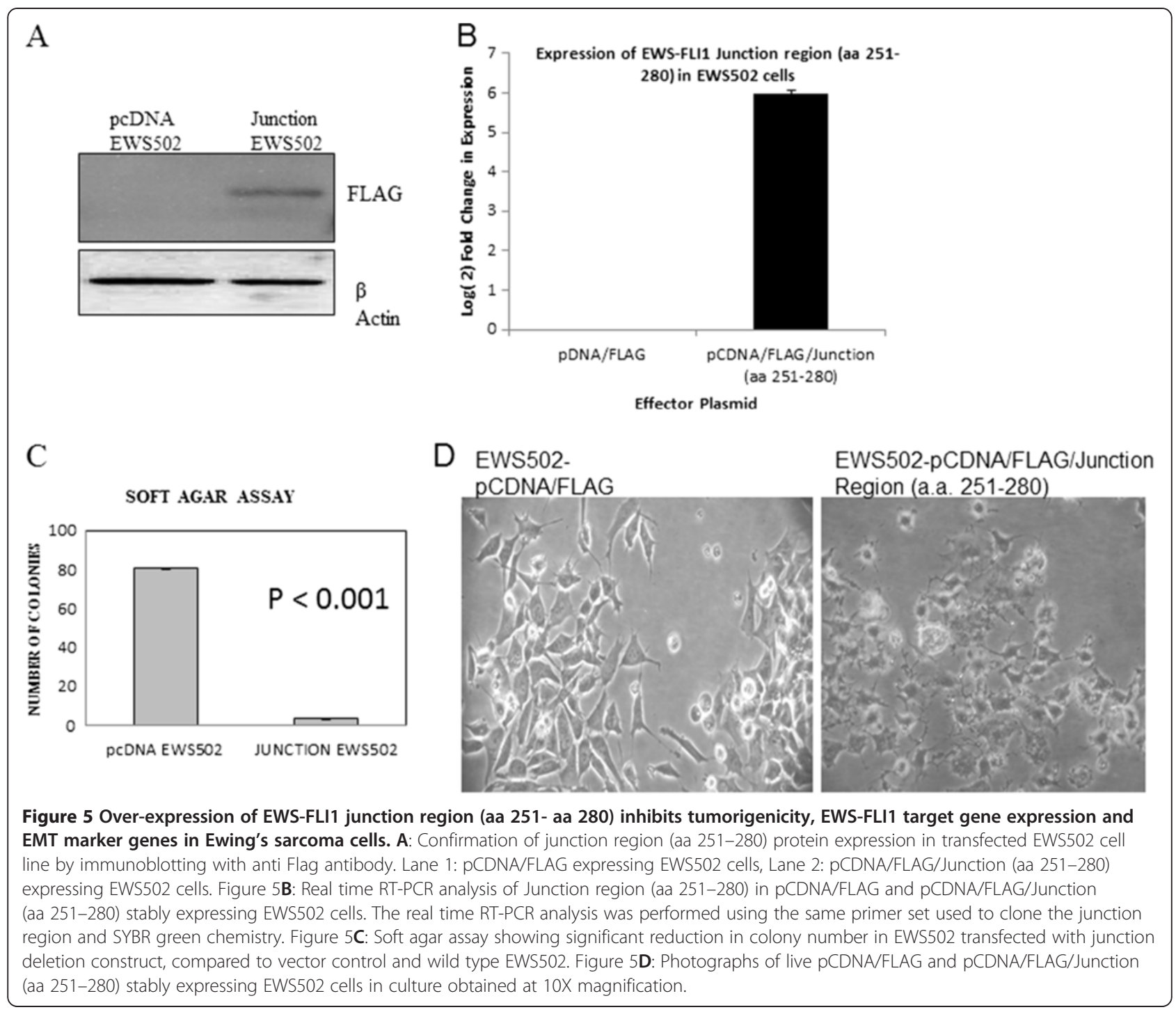

regulated target genes in junction construct transfected EWS502 cell line. EWS-FLI1 knockdown based studies and transcriptional profiling data [22] of various Ewing's sarcoma cell lines have shown that gene like NR0B1, NKX2.2, EZH2, GLI1 are up regulated by EWS-FLI1. Functional studies revealed that ongoing expression of these genes are required for the transformed phenotype of Ewing's sarcoma and reduction of any of these genes either in transcript level or in protein levels resulted in a significant reduction of oncogenic transformation in various Ewing's sarcoma cells [23-26]. This is in agreement with our results. In the present study we found more than 2 folds down regulation of NR0B1, NKX2.2 and GLI1 in EWS502 cell line in the presence of junction construct (compared to vector control) which could be the probable reason for the reduced tumorigenicity of junction construct transfected EWS502 cell line in vitro.
Previous studies have shown that TGF $\beta$ RII is a major down regulated target of EWS-FLI1 oncoprotein [27] and introduction of normal TGF $\beta$ RII into Ewing's sarcoma cell lines restores TGF $\beta$ sensitivity and blocks tumorigenicity. We observed a significant up regulation in TGF $\beta$ RII and KLF2 in the junction construct transfected EWS502 cells, which again supports the reduced tumorigenicity of junction construct transfected EWS502 cell line.

Earlier studies have shown that Ewing's sarcomaspecific EWS-ETS oncoproteins were capable of activating Cyclin D1 promoter in transient transfections of tissue culture cells $[28,29]$. Strong oncogenes such as c-MYC and Cyclin D1 appear to be up regulated by EWS-FLI1 in some models [30,31]. In our study we found 2.4 folds down regulation of Cyclin D1 and 1.48 fold down regulation of c-MYC in junction construct transfected EWS502 compared to vector control. 


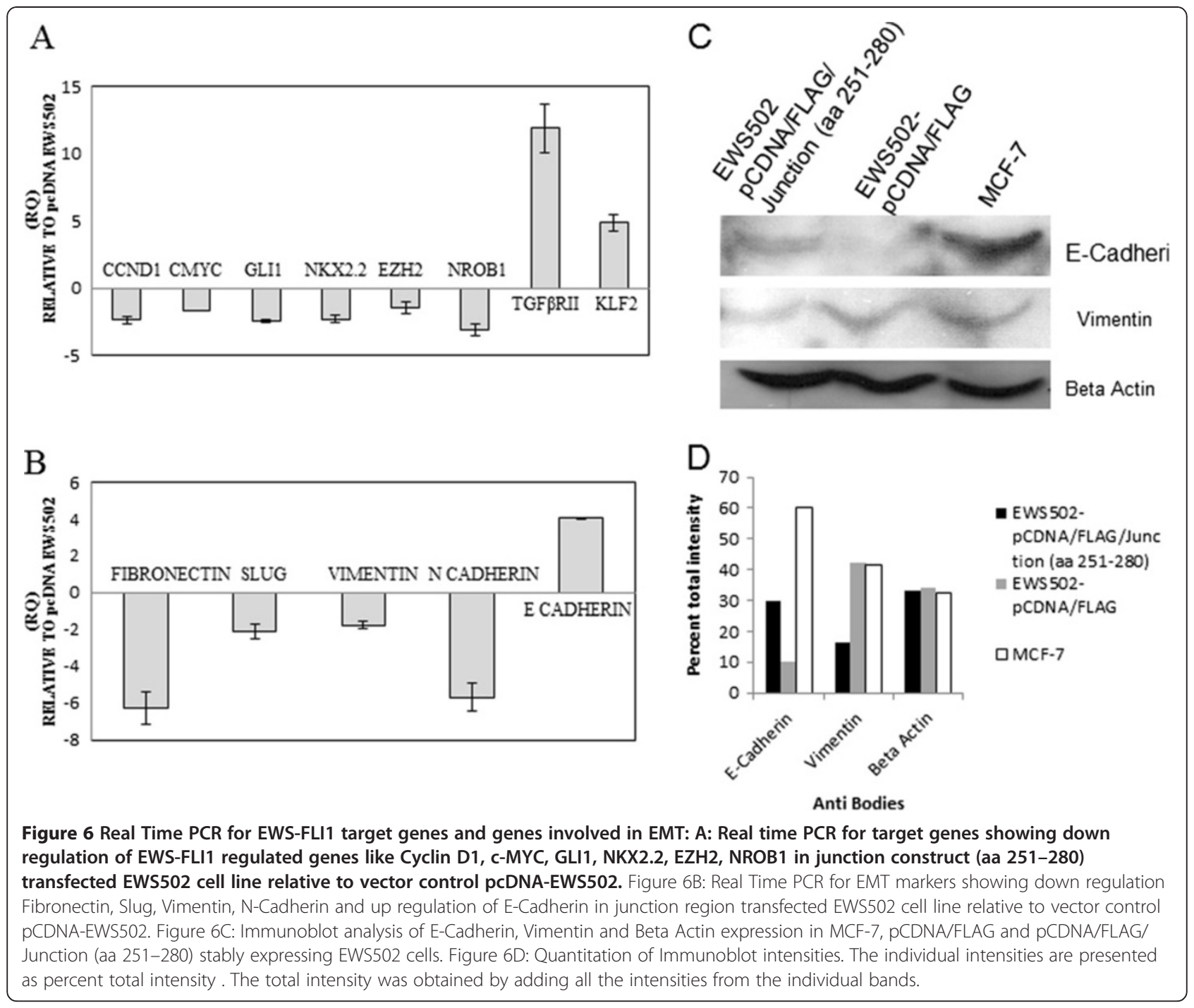

Imunohistochemistry based studies on EMT markers in earlier studies have shown that Ewing's sarcoma cells are positive for Vimentin [32]. Ewing's sarcoma cell lines produce a complex extra cellular matrix containing Fibronectin [33]. Previous studies have shown that Slug expression triggers EMT and its expression is inversely correlated with E-Cadherin expression [34]. In the present work we have suggested that down regulation of mesenchymal markers and up regulation of epithelial marker like E-Cadherin in EWS502 cell line, in the presence of junction construct may be a probable reason for the suppression of tumorigenicity in vitro.

\section{Conclusions}

We for the first time have identified a region encompassing the junction region of EWS-FLI1 protein to be part of putative small molecule binding sites (aa 251 - 343) and this region can be expressed in the soluble form which could pave the way for structural characterization of this region. Our study also indicates a propensity for a short junction construct (aa $251-280$ a 30 amino acid-peptide) to exhibit a dominant negative effect on the functions of wild type EWS-FLI1 protein by inhibiting target gene expression and tumorigenicity in vitro.

\section{Competing interests}

Authors declare no conflict of interest.

\section{Authors' contributions}

BJ performed the cloning and molecular genetic studies and drafting of the manuscript, RV performed sequence and bio-informatics analysis, GG performed the Western blotting and revision of the manuscript, KS performed the in-silico structural analysis, and TR conceptualized the study, analyzed the data and drafted and revised the manuscript. All authors read and approved the final manuscript.

\section{Acknowledgements}

The study was funded by Department of Science and Technology (DST), Govt. of India. EWS502 Ewing's sarcoma cell line is a generous gift from Dr Richard Smith, University of Utah, USA. Dr. N. Gautam, Dr. Karthi, Faculty, Centre of Advanced Studies in Crystallography and Biophysics, University of 
Madras, Chennai and Molecular Biophysics Unit, IISc, Bangalore are gratefully acknowledged for their help in carrying out CD experiments.

Received: 24 November 2011 Accepted: 30 October 2012 Published: 12 November 2012

\section{References}

1. Riggi N, Stamenkovic I: The Biology of Ewing sarcoma. Cancer Lett 2007, 54:1-10.

2. Arvand A, Denny CT: Biology of EWS/ETS fusions in Ewing's family tumors. Oncogene 2001, 20:5747-5754.

3. Hany MA, Adams W, Moos R, Betts DR, Niggli FK, Briner J: Modern diagnostic methods in the Ewing's sarcoma family: Six patients with histologic soft tissue tumors. Mol Diagnosis 1997, 2:15-22.

4. Delattre O, Zucman J, Plougastel B, Desmaze C, Melot T, Peter M, Kovar H, Joubert I, de Jong P, Rouleau G: Gene fusion with an ETS DNA-binding domain caused by chromosome translocation in human tumours. Nature 1992, 359:162-165.

5. Jedlicka P: Ewing sarcoma, an enigmatic malignancy of likely progenitor cell origin, driven by transcription factor oncogenic fusions. Int J Clin Exp Pathol 2010, 3:338-347.

6. May WA, Lessnick SL, Braun BS, Klemsz M, Lewis BC, Lunsford LB, Hromas R, Denny CT: The Ewing's Sarcoma EWS/FLI-1 Fusion Gene Encodes a More Potent Transcriptional Activator and Is a More Powerful Transforming Gene than FLI-1. Mol Cell Biol 1993, 13:7393-7398.

7. May WA, Gishizky ML, Lessnick SL, Lunsford LB, Lewis BC, Delattre O, Zucman J, Thomas G, Denny CT: Ewing sarcoma 11;22 translocation produces a chimeric transcription factor that requires the DNA-binding domain encoded by FLI1 for transformation. Proc Natl Acad Sci 1993, 90:5752-5756.

8. Ohno T, Rao VN, Reddy ES: EWS/Fli-1 Chimeric Protein Is a Transcriptional Activator1. Cancer Res 1993, 53:5859-5863.

9. Dunker AK, Garner E, Guilliot S, Romero P, Albrecht K, Hart J, Obradovic Z, Kissinger C, Villafranca JE: Protein disorder and the evolution of molecular recognition: theory, predictions, and observations. Pacific Symp Biocomputing 1998, 3:473-484.

10. Dunker AK, Obradovic Z, Romero P, Garner EC, Brown CJ: Intrinsic protein disorder in complete genomes. Genome Inform Ser Workshop Genome Inform 2000, 11:161-171.

11. Davis GD, Elisee C, Newham DM, Harrison RG: New fusion protein systems designed to give soluble expression in Escherichia coli. Biotechnol Bioeng 1999, 65:382-388.

12. Nilsson J, Stahl S, Lundeberg J, Uhlen M, Nygren PA: Affinity Fusion Strategies for Detection, Purification and Immobilization of Recombinant Proteins. Prot Exp Purif 1997, 11:1-16.

13. Foldlndex@ [http://bioportal.weizmann.ac.il/fldbin/findex] Prilusky J.

14. Prilusky J, Felder CE, Zeev-Ben-Mordehai T, Rydberg EH, Man O, Beckmann JS, Silman I, Sussman JL: Foldlndex: a simple tool to predict whether a given protein sequence is intrinsically unfolded. Bioinformatics 2005, 21:3435-3438

15. Uren A, Toretsky JA: Ewing's sarcoma oncoprotein EWS-FLI1: the perfect target without a therapeutic agent. Future Oncol 2005, 1:521-528.

16. Uren A, Tcherkasskaya O, Toretsky JA: Recombinant EWS-FLI1 Oncoprotein Activates Transcription. Biochemistry 2004, 43:13579-13589.

17. Dunker AK, Brown CJ, Lawson J, lakoucheva LM, Obradovic Z: Intrinsic disorder and protein function. Biochemistry 2002, 41:6573-6582.

18. Toretsky JA, Erkizan V, Levenson A, Abaan OD, Parvin JD, Cripe TP, Rice AM, Lee SB, Uren A: Oncoprotein EWS-FLI1 Activity Is Enhanced by RNA Helicase A. Cancer Res 2006, 66:5574-5581.

19. Jaulin LM, Masutani H, Robin P, Lipinski M, Bellan AH: SRE elements are binding sites for the fusion protein EWS-FLI-1. Nucleic Acids Res 1996, 24:1052-1058.

20. Bailly RA, Bosselut R, Zucman J, Cormier F, Delattre O, Roussel M, Thomas G, Ghysdael J: DNA-binding and transcriptional activation properties of the EWS-FLI-1 fusion protein resulting from the $t(11 ; 22)$ translocation in Ewing sarcoma. Mol Cell Biol 1994, 14:3230-3241.

21. Hancock JD, Lessnick SL: A transcriptional profiling meta-analysis reveals a core EWS-FLI gene expression signature. Cell Cycle 2008, 7:250-256.

22. Kinsey M, Smith R, Lessnick SL: NROB1 Is Required for the Oncogenic Phenotype Mediated by EWS/FLI in Ewing's Sarcoma. Mol Cancer Res 2006, 4:851-859.
23. Joo J, Christensen L, Warner K, States L, Kang HG, Vo K, Lawlor ER, May WA GLI1 Is a Central Mediator of EWS/FLI1 Signaling in Ewing Tumors. PloS One 2009, 4e7608.

24. Richter GHS, Plehm S, Fasan A, Rossler S, Unland R, Baiti IMB, Hotfilder M, Lowel D, Luettichau IV, Mossbrugger I, Martinez LQ, Kovard H, Staege MS, Tidow CM, Burdach S: EZH2 is a mediator of EWS/FLI1 driven tumor growth and metastasis blocking endothelial and neuro-ectodermal differentiation. Proc Natl Acad Sci 2009, 106:5324-5329.

25. Smith R, Owen LA, Trem DJ, Wong JS, Whangbo JS, Golub TR, Lessnick SL: Expression profiling of EWS/FLI identifies NKX2.2 as a critical target gene in Ewing's sarcoma. Cancer Cell 2006, 9:405-416.

26. Hahm KB, Cho K, Lee C, Im YH, Chang J, Choi SG, Sorensen PHB, Thiele CJ, Kim SJ: Repression of the gene encoding the TGF- $\beta$ type II receptor is a major target of the EWS-FLI1 oncoprotein. Nat Genet 1999, 23:222-227.

27. Zhang J, Hu S, Schofield DE, Sorensen PHB, Triche TJ: Selective usage of D-type cyclins by Ewing's tumors and rhabdomyosarcomas. Cancer Res 2004, 64:6026-6034.

28. Fuchs B, Inwards CY, Janknecht R: Vascular Endothelial Growth Factor Expression is Up-Regulated by EWS-ETS Oncoproteins and Sp1 and May Represent an Independent Predictor of Survival in Ewing's Sarcoma. Clin Cancer Res 2004, 10:1344-1353.

29. Fukuma M, Okita H, Hata Jl, Umezawa A: Upregulation of Id2, an oncogenic helix-loop-helix protein, is mediated by the chimeric EWS/ets protein in Ewing sarcoma. Oncogene 2003, 22:1-9.

30. Dauphinot L, De Oliveira C, Melot T, Sevenet N, Thomas V, Weissman BE, Delattre O: Analysis of the expression of cell cycle regulators in Ewing cell lines: EWSFLI-1 modulates p57KIP2 and c-Myc expression. Oncogene 2001, 20:3258-3265.

31. Lucas DR, Bentley G, Dan ME, Tabaczka P, Poulik JM, Mott MP: Ewing Sarcoma vs Lymphoblastic Lymphoma: A Comparative Immunohistochemical Study. Am J Clin Pathol 2001, 115:11-17.

32. Scarpa S, D'Orazi G, Modesti M, Modesti A: Ewing's sarcoma lines synthesize laminin and fibronectin. Pathol Anat Histopathol 1987 410:375-381.

33. Mancera PAP, Herrero IG, Caro MP, Cianca NG, Flores T, Adan AG, Pintado B, Martın MS, Garcia IS: SLUG in cancer development. Oncogene 2005, 24:3073-3082.

34. Bolós V, Peinado H, Moreno MAP, Fraga MF, Esteller M, Cano A: The transcription factor Slug represses E-cadherin expression and induces epithelial to mesenchymal transitions: a comparison with Snail and E47 repressors. J Cell Sci 2003, 116:499-511.

\section{doi:10.1186/1471-2407-12-513}

Cite this article as: Jully et al:: Junction region of EWS-FLI1 fusion protein has a dominant negative effect in Ewing's Sarcoma in vitro. BMC Cancer 2012 12:513.

\section{Submit your next manuscript to BioMed Central and take full advantage of:}

- Convenient online submission

- Thorough peer review

- No space constraints or color figure charges

- Immediate publication on acceptance

- Inclusion in PubMed, CAS, Scopus and Google Scholar

- Research which is freely available for redistribution 